\title{
Der Akzent im Erza-Mordwinischen
}

Seit H. Paasonen seine immer noch unersetzliche Untersuchung "Mordvinische Lautlehre" (1893 als Dissertation und 1903 mit Berichtigungen ergänzt als MSFOu XXII) veröffentlichte, hat man über die Akzentuierung im Erza-Mordwinischen nichts wesentlich Neues vorbringen können. Vor allem durch zahlreiche Untersuchungen, durchgeführt von geborenen Mordwinen, sind Paasonens Ergebnisse in wertvoller Weise bestärkt worden, die abschliessende Feststellung nämlich, dass von einem festen System keine Rede sein kann, denn der Akzent ist im Prinzip wirklich frei. Die Wörter haben eigentlich keinen festen Akzent, jede beliebige Silbe kann betont werden und diese Betonung kann - was am symptomatischsten ist - abhängig von Situation und Sprecher fast willkürlich schwanken. Paasonen stellt fest: "Ich muss darauf verzichten, irgend welche näheren regeln für die betonung im ersa-dialect aufzustellen.» Ferner führt er an, die Wörter würden in Satz oft zu einem Redetakt verbunden, doch auch wenn ein Wort einen ganzen Redetakt ausfülle, werde es einmal so, einmal so betont. Aufschlussreich und gleichzeitig überzeugend war der Versuch, den Paasonen im Dorf Maresevo anstellte. Die schreibkundigen Mordwinen, die das Russische natürlich ausgezeichnet beherrschten, vermochten ohne Schwierigkeit im russischen Text die Betonung an der richtigen Stelle zu bezeichnen, während sie bei den mordwinischen Texten "beständig dabei auseinander gingen". Dies ist ausschliesslich so zu deuten, dass die Akzentuierung im Erza-Mordwinischen wirklich frei ist, sie wechselt auch bei ein und demselben Sprecher mit der jeweiligen Sprechsituation.

Wie bereits erwähnt, sind Paasonens genaue Beobachtungen durch spätere Untersuchungen bedeutend bestärkt worden. Für die einzelnen Dialekte sind etwas unterschiedliche Tendenzen 
festgestellt worden. Die beachtlichste Beobachtung ist vielleicht die, dass die Betonung allgemein vor allem beim einzelnen Wort die erste Silbe bevorzugt. Ferner lässt sich in verschiedenen Dialekten eine summarische Stabilisierung des Akzents für eine bestimmte Silbe nachweisen, z.B. in der 3.P. der zweisilbigen Verben auf der ersten oder zweiten Silbe, bei einzelnen Infinitivformen auf bestimmten Silben usw.

In den publizierten Sprachproben ist der Akzent meist nicht eingetragen. Wo das geschehen ist, wird Paasonens Resultat nur bestärkt. Das gilt auch für einige alte Evangelienübersetzungen, wo die Betonung bezeichnet ist.

Ernst Lewy hat sich sehr gründlich mit den Texten beschäftigt, die in A. A. ŠAChMatovs »Mordovskij Etnografičeskij Sbornik» enthalten und mit Betonungszeichen versehen sind. Šachmatov selbst stellt a.a.O. 759ff. fest, dass der Akzent im Dialekt von Suchoj Karbulak durchaus nicht immer auf der ersten Silbe liegt, wie sein wichtigster Informant behauptete, sondern im Satzzusammenhang oft auch auf anderen Silben. In seinem Aufsatz FUF XXIV S. 232-245 untersucht Lewy Šachmatovs Texte genauer und kommt zu dem Ergebnis, dass »die satzbetonung auch wieder für die betonung des wortes sehr wichtig ist». In seiner umfangreichen Abhandlung "Zur Betonung des Erdzja-Mordwinischen von Orkino" (1961) kommt er erneut auf die Frage zurück und analysiert Šachmatovs Texte anerkennenswert gründlich. Leider gibt die Arbeit nicht viel her; Lewy stellt wieder einmal fest, dass im Dialekt von Orkino die Wörter als solche keinen festen Akzent haben, sondern diesen erst im Satzzusammenhang erhalten.

Es ist nur natürlich, dass sich die Forscher durch eine solche fast vollständige Anarchie der Betonung vor den Kopf geschlagen fühlten. Gründe hat man eigentlich überhaupt nicht vorbringen können. Paasonen dachte offenbar einmal daran, eine derart unbestimmte Akzentuierung wie im Erza-Mordwinischen könne direkt von der finnisch-ugrischen Ursprache herrühren (s. a.a.O. IX). V. I. Lytkis hat ebenfalls angenommen, dass die fiu. Ursprache einen völlig freien Akzent gehabt hätte, s. ALH XX S. 245ff.

Durch Erkki Itkonen sind die Gedanken von der freien 
Akzentuierung der fiu. Ursprache schlagend widerlegt worden, s. FUF XXXIX S. 73. Was nun ausdrücklich das Erza-Mordwinische anbelangt, so dürfte es ziemlich klar sein, dass dessen Vertretung erst aus der dem Urmordwinischen folgenden Zeit stammt, also relativ jung ist. Die Erforscher des Mordwinischen sind sich weitgehend einig darin, dass das Mokscha den ursprünglichen Verhältnissen näher steht als das Erza. Bestimmte Lauterscheinungen im Erza sind kaum anders erklärlich. Paasonen, auf den dieser Gedanke zurückgeht, hat ihn - mit Ausnahme der obigen Bemerkung - auch später noch bestärkt, s. FUF XIII Anz. 23.

Eine bestimmte Ursache hat natürlich das Erza-Mordwinische in eine solche eigenartige Situation gebracht, die von dem recht klaren System abweicht, das im Mokscha beibehalten ist. Offensichtlich durch denselben Faktor gelangten auch volle Vokale in unbetonte Silben in Fällen, wo im Mokscha weiterhin die reduzierten $\hat{\partial}$ und $a$ begegnen, denn diese Dinge sind untrennbar mit der Akzentuierung verbunden.

Alle Versuche, im Erza selbst die Gründe für diese Entwicklung zu finden, die ja das gesamte Lautsystem weitgehend beeinflusst hat, scheinen aussichtslos. Um regelrechte Lautveränderungen kann es sich ja nicht handeln. Obgleich die eigentlichen, speziell ausserhalb des Mokscha-Einflusses stehenden Erzadialekte übereinstimmend die Entwicklung von reduzierten Vokalen in der nichtersten Silbe zu Vollvokalen aufweisen, hat diese Entwicklung doch zu recht verschiedenen Endergebnissen geführt. Nur die Schriftsprache hat die konsequente $o, e$-Vertretung, in den Mundarten steht mal $u$, $i$, mal $o$, $e$, die ausserdem untereinander ganz verschieden wechseln. Es ist also zu folgern, dass die Entwicklung der Vollvokale aus reduzierten Vokalen keine urerzanische Erscheinung sein kann. Es ist vielmehr das Ergebnis einer Parallelentwicklung, die wiederum sicher auf einen gemeinsamen Faktor zurückgeht. Natürlich muss vorausgesetzt werden, dass schon im Urmordwinischen die reduzierten Vokale in verschiedenen Umgebungen unterschiedliche Nuancen erhielten.

Ich habe zu keinem anderen Resultat kommen können, als dass dieser gemeinsame Faktor ausserhalb des Erza gesucht 
werden muss, denn um eine urmordwinische Disposition kann es sich nicht handeln. Dieser äussere Faktor kann meiner Ansicht nach nur die russische Sprache sein. Dadurch würde ja auch erklärt, warum Erza und Mokscha sich nicht in derselben Richtung entwickelt haben. Schliesslich war das Erza ja einem viel stärkeren russischen Einfluss ausgesetzt, und die Impulse kamen ausserdem zu einem beachtlichen Teil aus verschiedenen Quellen. Und doch ist es sehr schwer, diesen Gedanken überzeugend zu begründen. An sich ist es leichter, ihn abzulehnen, da man zu einer negativen Einstellung kaum Begründungen braucht. Wie ich habe feststellen können, hat jedoch J. D. Polivanov die Behauptung vorgebracht, dass das Russische wirklich die Ursache wäre für die heutige Situation im Erza-Mordwinischen. Leider existieren seine Ausführungen nur im Manuskript. T. A. IsAJEvs, die die Handschrift in ihrer Arbeit $\gg \mathrm{Ob}$ udarenii $\mathrm{v}$ erzja-mordovskom jazyke» erwähnt, kritisiert diese Ansicht mit der Begründung, dass das Russische auch das Akzentsystem anderer Sprachen nicht in dieser Weise störend beeinflusst habe. Erkki Itkonen scheint diese Kritik zu akzeptieren und vermutet seinerseits, es handle sich um das Ergebnis der Eigenentwicklung des Erza, wodurch die einzelnen Silben im Wort eine gleichwertige Stellung erhielten. Das Erza sei also einen Schritt weiter gegangen als Ungarisch und Finnisch, wo sich der Vokalismus der ersten und folgenden Silben von der ursprünglichen Unterschiedlichkeit in Richtung auf eine qualitative Gleichheit entwickelte, s. FUF XXXIX S. 74.

Dass für keine andere Sprache ein entsprechender Einfluss des Russischen nachgewiesen werden kann, ist als Argument praktisch hinfällig. Es existieren ja nur ganz wenige Sprachen, wo die Tiefe des russischen Einflusses auch in anderer Hinsicht mit dem verglichen werden kann, was im Erza feststellbar ist.

Ein gültiger Vergleichspunkt für die Entwicklung, die sich im Erza eindeutig vollzogen hat, ist mir aus der Geschichte anderer Sprachen nicht bekannt. Der Hinweis auf die französische Sprache genügt nicht (s. Lewy, a.a.O. S. 108 Fussn.), obgleich einige interessante Übereinstimmungen festzustellen sind, s. z.B. JeSPERSEN "Lehrbuch der Phonetik» S. 223. Die Ausgangspunkte sind völlig anders, und die İbereinstim- 
mungen nichts anderes als allgemeinphonetische Parallelerscheinungen. Oft kann ja eine ganz unterschiedliche historische Entwicklung verschiedenerseits zu einem gleichen Endergebnis führen; von einem gleichen Endergebnis aber braucht in diesem Zusammenhang überhaupt nicht gesprochen zu werden.

Um etwas Klarheit zu bekommen in diesen wirren Knäuel habe ich mir zwei Fragen gestellt.

Erstens: Wir wissen aus der Geschichte der Sprachen Europas gut, wie leicht die Akzentsysteme einander beeinflussen können. Nehmen wir an, dass das Russische wirklich auf die Entstehung der Verhältnisse im Erza eingewirkt hat. Wie hätte dies überhaupt möglich sein können? Im Russischen kann der Akzent prinzipiell auf jeder beliebigen Silbe liegen, doch ist es sehr wichtig, dass der Akzent in den Wortformen immer an dieselbe Silbe gebunden ist, z.B. Nom. ногá, Akk. нóгу. Die Dialekte können natürlich voneinander abweichen und vielleicht tritt mitunter in ein und derselben Mundart auch eine alternative Akzentuierung auf, doch ist der Akzent monoton und das macht ihn auch phonematisch relevant, z.B. му́ка мука́ руки́ $\sim$ ру́ки, usw. Ein solches System kann nicht auf eine andere Sprache übergehen, die in der Struktur völlig verschieden ist. Im Erza-Mordwinischen hat ja der Akzent - wie stets festgestellt - keine phonematische Funktion, er ist wirklich auch in dieser Hinsicht frei. Im Urmordwinischen lag der Akzent allgemein immer auf der ersten silbe, konnte unter bestimmten Bedingungen aber auch auf eine der folgenden Silben übergehen, wenn in ihnen ein sonores a stand. Die russische Sprache konnte jedoch dazu beitragen, das Erza von einer zu starken Gebundenheit an die Betonung der ersten Silbe zu befreien.

Ein sehr wichtiger Umstand ist der dynamische Charakter des Akzents. Uns sind jene Dialekte noch nicht genügend bekannt, die speziell auf das Erza eingewirkt haben. Auf jeden Fall waren es Okanje-Dialekte. Die Lehnwörter im Mokscha weisen wiederum auf den Einfluss von Akanje-Dialekten hin und es ist festzustellen, dass in diesen Dialekten die Unterschiede zwischen betonten und unbetonten Silben recht beachtlich und auch die qualitativen Differenzen der Vokale deutlich sind. In 
den Akanje-Dialekten ist die Anzahl der Phoneme in unbetonten Silben kleiner als in betonten. In den Okanje-Dialekten ist die Situation eine andere. Sowohl die betonten als auch die unbetonten Silben verfügen über dieselbe Anzahl von Phonemen: $a, e, i, o, u$. Ausserdem ist in zahlreichen Okanje-Dialekten der dynamische Unterschied zwischen betonter und unbetonter Silbe recht gering. Ich nehme an, dass die russischen Dialekte, die den Anstoss gaben zur Entwicklung der reduzierten Vokale zu Vollvokalen in den Erza-Mundarten, gerade solche Dialekte waren, in denen es keine grossen Unterschiede gab zwischen betonter und unbetonter Silbe.

Dies alles ist natürlich wichtig, erklärt aber noch nicht die deutliche Systemlosigkeit der erzamordwinischen Akzentuierung. Wir brauchen also weitere Argumente.

$\mathrm{Z}$ w e i t e n s: Gibt es andere Erscheinungen, die von einem störenden Einfluss des russischen auf das mordwinische Lautsystem zeugen, von Besonderheiten, die in keiner Weise als Ergebnis einer regelmässigen Lautentwicklung hingestellt werden können? Diese Frage lässt sich m.E. relativ leicht beantworten und ich brauche nicht alle Phänomene anzuführen, sondern begnüge mich mit dem vielleicht offensichtlichsten. Schon recht frühzeitig hat man mit soliden Begründungen gefolgert, dass im Urmordwinischen im Wortanlaut keine stimmhaften Klusile und Sibilanten gestanden haben. $\$ 13$ der »Mordvinischen Lautlehre" von Paasonen lautet: "Das gemeinmordvinische hat im wortanlaut sicherlich nur die stimmlosen verschluss- und reibelaute $k, p, t, s, \breve{s}$ gekannt.» Dieser Stand hat sich noch in einigen Mokscha-Dialekten erhalten, die nicht dem stärksten russischen Druck ausgesetzt waren, wie z.B. zur Zeit von Paasonen im Dialekt von Pšenevo. Durch die zahlreichen Lehnwörter ist das alte System jedoch allmählich durchbrochen worden. Zuerst wurden natürlich die im Anlaut stehenden stimmhaften Laute des Russischen wie auch Tatarischen durch die entsprechenden stimmlosen ersetzt, z.B. mSel. šuba $n$ 'hölzerne Kanne' < russ. жбан, erz.Mar. šagala 'Brenneisen' $<$ russ. жигало, mSel. pur'kän' 'Mohrrübe' < russ. буркан.

Bald lernt man jedoch auch im Wortanlaut stimmhafte Laute, und nun folgen sehr interessante Erscheinungen. Sehr 
allgemein erhält ein im Russischen im Wortanlaut stimmloser Laut eine stimmhafte Entsprechung, z.B. bau.k 'Spinne' < russ. паук, gleichsam als sei das Wort auf diese Weise besser gezeichnet. Allmählich dringt der stimmhafte Laut dann auch in den bodenständigen Wortschatz ein und erobert ganz offensichtlich vor allem die Wörter, die deskriptiv sind oder anderweitig diverse Gefühlswerte enthalten. Ein ursprünglich fremder Laut hat eine eigene Affektfunktion erhalten, wodurch die regelmässige Lautentwickelung verdrängt wurde. Diese Fragen habe ich ausführlicher behandelt in meinem Artikel "IntenseForms and Sound System» FUF XXXI S. 133-148.

Von diesen Tatsachen ausgehend kann m.E. auch das Akzentsystem des Erza - oder besser die Systemlosigkeit - leichter verstanden werden. Von einer lauthistorischen Entwicklung kann ja keine Rede sein. Wenn das Russische diese Erscheinung entstehen liess, dann in erster Linie durch Vermittlung seiner zahlreichen Lehnwörter und teilweise natürlich auch durch den Einfluss neuer Artikulationsgewohnheiten bei Zweisprachigen.

Ich möchte den Entwicklungsverlauf folgendermassen schildern. Die russischen Lehnwörter passen sich in ihren Akzentverhältnissen dem Dialekt des Entlehnenden an; die Wörter also, die auf der zweiten oder darauf folgenden Silbe betont sind, erhalten den Ton auf der ersten Silbe. Paasonen weist a.a.O. IX Fussn. die von Wiedemann in seiner Grammatik (S. 15-16) vorgebrachte Behauptung zurück, die aus dem Russischen und Tatarischen entlehnten Wörter würden ihre ursprüngliche Betonung beibehalten. Was speziell das Mokscha anbelangt, so entspricht die Mehrheit der russischen Lehnwörter heute der Akzentuierung im Russischen, doch gibt es eine grosse Anzahl von Wörtern, die sich völlig dem eigenen System des Mokscha angeglichen haben, s. Paasonen a.a.O. S. 116. Eine entsprechende Feststellung bringt auch JENő JuHÁsz, "Moksamordvin szójegyzék» S. 20.

Ich sehe die Dinge so, dass im Mokscha die Entwicklung hier stehen blieb: das russische Lehnwort hat sich entweder dem eigenen System angepasst oder der Akzent folgt dem Original. Im Erza dagegen waren Doppelformen durchaus möglich, weil es keinen grundsätzlichen Unterschied gab zwischen betonter 
und unbetonter Silbe, da sich die dynamischen und qualitativen Differenzen ausgeglichen hatten. Im Mokscha hat das ja nicht vor sich gehen können, da dessen Entwicklung dieselben Bahnen nicht so weit gegangen ist wie das Erza. Da die Akzentuierung der Lehnwörter recht willkürlich wurde, übertrug sich dieselbe Erscheinung allmählich auch auf den eigenständigen Wortschatz. Vor allem in emotionalen Zusammenhängen verschob sich der Akzent auf eine nichterste Silbe nach genau demselben Prinzip wie bei den stimmhaften Lauten im Wortanlaut. Geltend macht sich ferner der Satzrhythmus - leicht beeinflusst von einer mehr oder minder ausgeprägten Affektgeladenheit wodurch die allgemeine Färbung der Rede immer mehr überwiegend russischen Charakter annehmen kann.

Ich bin mir natürlich darüber im Klaren, dass diese Überlegungen keine endgültig bindende Kraft besitzen. Es ist noch viel detaillierte Forschungsarbeit zu leisten. Da ich jedoch andererseits davon überzeugt bin, dass die hier vorgebrachten Aspekte bei der Lösung der Frage nicht ausser acht gelassen werden können, habe ich sie zu Papier gebracht.

Paavo Ravila 